\title{
An Efficient Medical Data Classification based on Ant Colony Optimization
}

\author{
Jyotsna Bansal \\ M. Tech Research scholar \\ CSE dept., BUIT \\ Bhopal, India
}

\author{
Divakar Singh \\ Head of CSE dept., BUIT \\ Barkatullah University, \\ Bhopal, India
}

\author{
Anju Singh \\ Asst. Prof. IT dept., BUIT \\ Barkatullah University, \\ Bhopal, India
}

\begin{abstract}
In the case of different diseases classification is an important aspect so that one can find the infected set efficiently. In this paper three different dataset named Leukemia, Lung Cancer and Prostate from the UCI machine learning repository are considered and apply efficient association based ant colony optimization for improving the classification accuracy. In our approach one can select the dataset. The data set has been refined according to the attributes. Then final data set is achieved on which we apply the next inabilities. The maximum threshold will be determined by finding the support value. So the support values are fetched and according to the support value, it will be categorized in two different parts that is relevant or irrelevant. In our case it is 0.5 . If the set crosses the maximum threshold then it will be qualify for the final set otherwise it is discarded. Then ACO mechanism has been applied on the final dataset to find the classification accuracy. Our results show the effectiveness of our approach.
\end{abstract}

\section{General Terms}

Leukemia, lung cancer, prostate

\section{Keywords}

Classification, Clustering, Feature selection, ACO

\section{INTRODUCTION}

Till now there are lot of studies had been made in the direction of classification and clustering like weighted voting (WV) [1], k-nearest neighbor (k-NN)[2], support vector machine (SVM) [3], and Fisher's linear discriminate analysis (LDA) [4] . but these are not effective for better predicting the cases like whether a patient has got a particular disease or not [5],[6][7].

There are several studies are done for data portioning, data management and way of retrieval [8][9][10]. Better classification is not achieved by a single algorithm but it can be possible if it can be done by an hybrid platform. There are several algorithms which are also using genetic and fuzzy set are also applied in these areas [11]. Combine the fuzzy and search capabilities of Genetic Algorithms (GAs) may improve the optimal fuzzy rule and improve the rule generation also [12].

Association based classifier are also used and provide some good utility. Data mining can be used for better retrieval of the data [13][14]. Means data mining can exploits the data in any environment so the data retrieval is easy. So then there is need of better optimization so that we can able to find the good rules which are showing the classification accurately. In this stage Ant Colony Optimization (ACO) technique can be used for this improvement [15][16].
The next sections are divided in the following manner. Literature Review are shown in section 2,proposed approach is shown in section 3,discuss the result analysis which in section 4,section 5 shows the conclusion and finally result analysis are given.

\section{LITERATURE REVIEW}

In [17] author discusses about DNA microarray technologies. They suggest that they are typically noisy and have very high dimensionality and these make the mining of such data for gene function prediction very difficult. So they proposes an incremental fuzzy mining technique called incremental fuzzy mining (IFM). Gene prediction will improve by different membership parameter with the help of IFM. In [18] there is a comparison between three methods which are K-Means with Signal to noise ratio (SNR) and other uses (SVM), probabilistic Neural Network (PNN) and Feed Forward Neural Network (FNN). The final results of two approaches they have got $100 \%, 96 \%$ and $96 \%$ accuracy with SVM, k-NN and PNN respectively in first approach with five numbers of genes. In [19] author suggests sample categorization of co regulated gene is an important task. In this regard they proposed a gene clustering algorithm to group genes from microarray data. It exactly incorporates the tip-off of reproduce categories in the orchestration action for purposefulness groups of co-regulated genes relating to foolhardy alliance to the mimic categories, yielding a supervised gene clustering algorithm. In [20] SVM was discussed for earning tasks which is useful in classification, regression or novelty detection. Author wants to improve the generalization problem. So based on the problem they use Particle swarm optimization (PSO) which is a population based stochastic optimization technique where the potential solutions, called particles, fly through the problem space by following the current optimum particles. They used Principal Component Analysis (PCA) for reducing features of breast cancer, lung cancer and heart disease data sets and an empirical comparison of kernel selection using PSO for SVM is used to achieve better performance. In [21] mutual information-based feature selection framework is used. In [22] clustering algorithms have been suggested to analyze genome expression data, but fewer solutions have been implemented to guide the design of clustering-based experiments and assess the quality of their outcomes. A collection wring setting provides insights into the organization of predicting the meticulous the number of clusters. In [23] suggest that genes are encoding regions that form essential building block within the cell and lead to proteins which are achieving various functions. In spite of that, different genes may be mutated befitting to formal or front deed data and this is a main cause for various diseases. So author suggested that it is important to identify mutated genes as disease 
biomarkers. They address this problem by introducing a comprehensive framework that incorporates the two stages of the process, namely feature selection and sample classification. and sure thing, lofty dimensionality in interpretation of the in the midst of genes and brief expanse of samples distinguishes gene parturition text as an create call for the trifling frame of reference Reducing the dimensionality is unembellished to efficiently analysis the samples for effective knowledge discovery.

\section{PROPOSED ALGORITHM}

In this approach, first select the data set as shown in figure 1. Based on the data set it is first categorize in two parts. In the first part the support greater than 0.5 are present and in the second part store the values which are less than 0.5. The selected support in the second part is first evaluated and optimize by ACO. If some patterns observed which are relevant according to the minimum support value. Then after the final iteration we will put those set in the first set. Then optimize the dataset by ACO again but now with the first part including the set which are found positive in the second part. Then better classification results will be achieved after 8 iteration which will be shown in the result section. The dataset had been taken from UCI machine learning repository.

\section{Algorithm: ACO based Classification}

Input: IS (is1, is2......isn)

Output: OS (sv1, sv3.....svn)

\section{Terminology:}

\section{NRS $\rightarrow$ Non relevant set \\ RS $\rightarrow$ Relevant Set \\ IS $\rightarrow$ Input Set \\ OS $\rightarrow$ Output Set \\ IT $\rightarrow$ Initial trail \\ $n \rightarrow$ Number of Agents \\ $\mathrm{EV} \rightarrow$ evaporation value \\ FS $\rightarrow$ Final set}

Step 1: IS $=\{i 1, i 2 \ldots$ in $\}$

Step 2: Apply the k-group on IS and form the representative block.

Step 3: First consider the NRS set

3a. Assign each values as nrs1, nrs2 ......nrsn.

3b. for $i=1$ to 5 do

$\mathrm{IT}=\sum \mathrm{nrs} 1+\mathrm{nrs} 2+\ldots . .+\mathrm{nrsn} / \mathrm{n}$

$\mathrm{EV}=\{0.2,0.4,0.6,0.8\}$

If $(\mathrm{i}==1)$

IT1 $=$ IT

If $(\mathrm{i}==2)$

IT2=IT-EV1(0.2)

If $(\mathrm{i}==3)$

IT3=IT-EV1 $(0.4)$

If $(\mathrm{i}==4)$

IT4=IT-EV1(0.6)

If $(\mathrm{i}==5)$

IT5=IT-EV1 $(0.8)$

If $\left(\mathrm{IT}_{\mathrm{n}}>\mathrm{IT}_{\mathrm{n}-1}\right)$

$\mathrm{IT}_{\mathrm{n}-1}=\mathrm{IT}_{\mathrm{n}}$
Step 4: if(nrs1,nrs2 ...nrsn $>=0.5)$

$\mathrm{RS}=\{\mathrm{rs} 1, \mathrm{rs} 2 \ldots \ldots \ldots \mathrm{nrs} 1 \ldots \ldots . \mathrm{nrsn}\}$

Step5: .for $\mathrm{i}=1$ to 8 do

$\mathrm{IT}=\sum \mathrm{nrs} 1+\mathrm{nrs} 2+\ldots . .+\mathrm{nrsn} / \mathrm{n}$

$\mathrm{EV}=\{0.2,0.4,0.6,0.8\}$

When moving from first neighbor to second neighbor node the agent can update the pheromone trails.

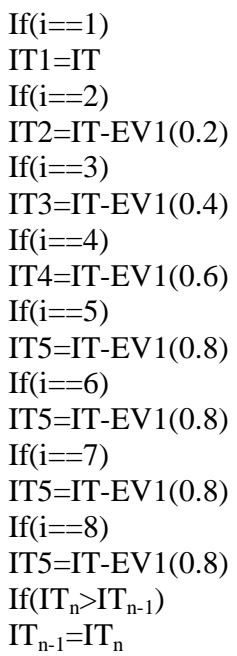

Step 6: The FS will be achieved and we can calculate the classification.

The above algorithm shows the whole working scenario of our proposed work. By this algorithm firstly divide the dataset into k parts. In our case it is 2. Then apply ACO in the irrelevant set and search for the relevancy. If any of the item set found relevant. It will be added in the relevant set. Then finally ACO will be applied on the relevant data set to find the accuracy.

The steps are following:

1) Initialize the initial weight of the agents.

2) Then each agent will start with some random assigned values.

3) Then we perform the summation of all the support value and divide it by the numbers which will be subtracted by the evaporation value.

4) If the new value is greater than the global optimum value then we replace the previous value with the updated value, otherwise it remain same.

If the value is greater than 1 or less than 0 then the previous value will be retain. 


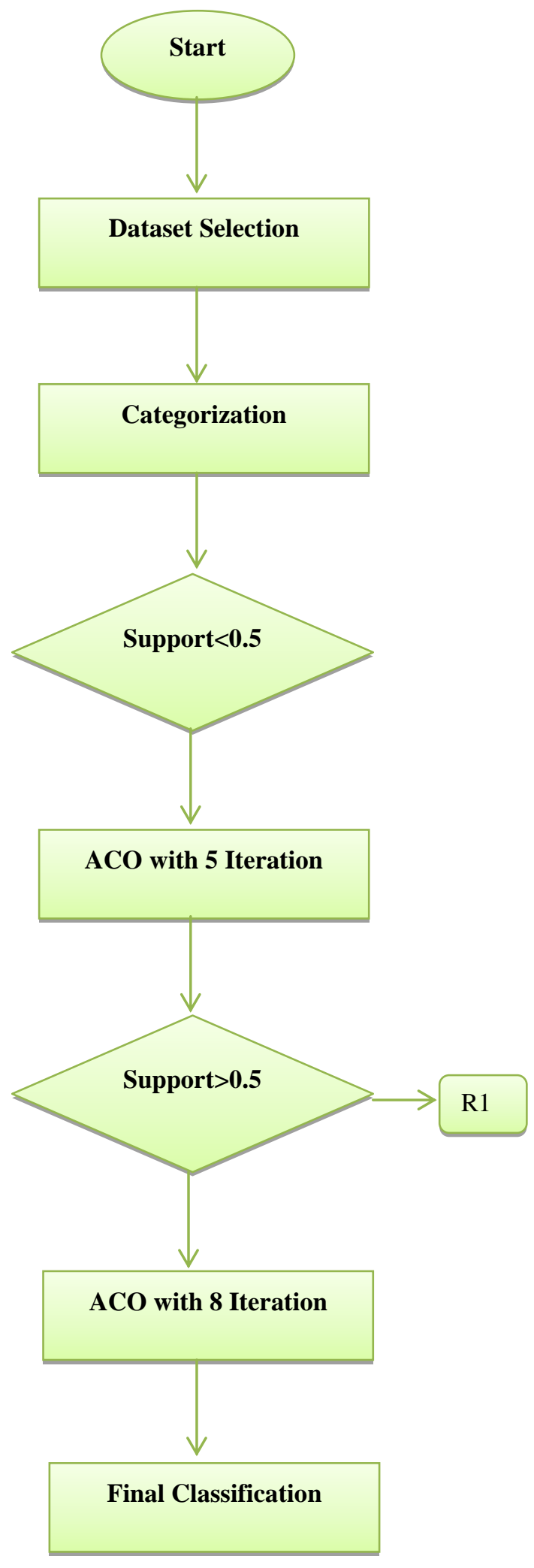

\section{RESULT ANALYSIS}

In our proposed approach three data set are used as shown in table 1 , table 2 and table 3 . Optimized format are shown in table 4 , table 5 and table 6 . The classification accuracy is shown in figure 2 and the overall accuracy is shown in figure 3. comparison with the existing techniques also shown in table 7. It shows the effectiveness of our approach.

The advantages apart from play go infirmity as a bequeathed plague at cellular equal is different from type to type. For holder, the correctness of colon gene habituated is further than others. Allow for, deriving near predisposed to gene sets company a insubordinate function, indebted shriek solitarily to text mining techniques but rather the relatively small number of samples available for the analysis

Table 1: Dataset1

\begin{tabular}{|c|c|c|c|c|c|c|c|c|c|c|}
\hline \multicolumn{11}{|c|}{ Prostate } \\
\hline A1 & $\mathbf{A 2}$ & A3 & A4 & A5 & A6 & A7 & A8 & A9 & A10 & A11 \\
\hline 1 & -0.579818495 & 2.769459 & 0.5 & \begin{tabular}{ll|}
1.38629436 \\
\end{tabular} & 0 & 1.38629436 & 6 & 0 & 0.4307829 & $\mathrm{~T}$ \\
\hline 2 & 0.223143551 & 3.244544 & 0.63 & \begin{tabular}{|l|}
1.38629436 \\
\end{tabular} & 0 & 1.38629436 & 6 & 0 & 1.047319 & $F$ \\
\hline 3 & 0.254642218 & 3.604138 & 0.65 & 1.38629436 & 0 & 1.38629436 & 6 & 0 & 1.2669476 & $\mathrm{~T}$ \\
\hline 4 & -1.347073648 & 3.598681 & 0.63 & 1.2669476 & 0 & 1.38629436 & 6 & 0 & 1.2669476 & $\mathrm{~T}$ \\
\hline 5 & 1.613429934 & 3.022861 & 0.63 & \begin{tabular}{|l|}
1.38629436 \\
\end{tabular} & 0 & -0.597837 & 7 & 30 & 1.2669476 & $\mathrm{~T}$ \\
\hline 6 & 1.477048724 & 2.998229 & 0.67 & 1.38629436 & 0 & 1.38629436 & 7 & 5 & 1.3480731 & $\mathrm{~T}$ \\
\hline 7 & 1.205970807 & 3.442019 & 0.57 & \begin{tabular}{|l|}
1.38629436 \\
\end{tabular} & 0 & 0.43078292 & 7 & 5 & 1.3987169 & F \\
\hline 8 & 1.541159072 & 3.061052 & 0.66 & \begin{tabular}{|l|}
1.38629436 \\
\end{tabular} & 0 & 1.38629436 & 6 & 0 & 1.446919 & $\mathrm{~T}$ \\
\hline 9 & -0.415515444 & 3.516013 & 0.7 & 1.24415459 & 0 & -0.597837 & 7 & 30 & 1.4701758 & $\mathrm{~T}$ \\
\hline 10 & 2.288486169 & 3.649359 & 0.66 & 1.38629436 & 0 & 0.37156356 & 6 & 0 & 1.4929041 & T \\
\hline
\end{tabular}

Table 2: Dataset2

\begin{tabular}{|c|c|c|c|c|c|c|c|c|c|c|}
\hline \multicolumn{11}{|c|}{ LEUKEMIA } \\
\hline Sample & ALL_AML & BM_PB & |TB_Cell & FAB & Date1 & Gender & Blast & Tratment & PS & Response \\
\hline 1 & ALL & BM & B-cell & & 09/04/96 & M & & & 1 & DFCI \\
\hline 2 & ALL & BM & T-cell & & & M & & & 0.41 & DFCI \\
\hline 3 & ALL & BM & T-cell & & & M & & & 0.87 & DFCI \\
\hline 4 & ALL & BM & B-cell & & & & & & 0.91 & DFCI \\
\hline 5 & ALL & BM & B-cell & & & & & & 0.89 & DFCI \\
\hline 6 & ALL & BM & T-cell & & & M & & & 0.76 & DFCI \\
\hline 7 & ALL & BM & B-cell & & $03 / 25 / 83$ & F & & & 0.78 & DFCI \\
\hline 8 & ALL & BM & B-cell & & & F & & & 0.77 & DFCI \\
\hline 9 & ALL & BM & T-cell & & & M & & & 0.89 & DFCI \\
\hline 10 & ALL & BM & T-cell & & 07/23/87 & M & & & 0.56 & DFCI \\
\hline
\end{tabular}

Fig 1: Flowchart 
Table 3: Dataset3

\begin{tabular}{|r||c|c||l|r|}
\hline \multicolumn{5}{|c|}{ Lung } \\
\hline ID & Field1 & Field2 & Field3 & Field4 \\
\hline \hline 1 & NODE1X & ARRY71X & ARRY63X & 0.9398193359375 \\
\hline \hline 2 & NODE2X & ARRY48X & ARRY42X & 0.880126953125 \\
\hline \hline 3 & NODE3X & ARRY25X & ARRY24X & 0.86590576171875 \\
\hline \hline 4 & NODE4X & ARRY66X & ARRY65X & 0.8321533203125 \\
\hline \hline 5 & NODE5X & ARRY2X & ARRY1X & 0.816650390625 \\
\hline \hline 6 & NODE6X & ARRY62X & ARRY61X & 0.8052978515625 \\
\hline \hline 7 & NODE7X & ARRY64X & NODE2X & 0.8026123046875 \\
\hline \hline 8 & NODE8X & ARRY59X & NODE7X & 0.7900390625 \\
\hline \hline 9 & NODE9X & ARRY15X & ARRY14X & 0.7698974609375 \\
\hline \hline 10 & NODE10X & ARRY47X & ARRY46X & 0.75811767578125 \\
\hline \hline
\end{tabular}

Table 4: Optimized From Dataset1

\begin{tabular}{|l|r|}
\multicolumn{2}{|c|}{ tmax1 } \\
\hline \multicolumn{1}{|c|}{ itemset } & \multicolumn{1}{c|}{ percentage } \\
\hline \hline A1 & 1 \\
\hline A3 & 0.9481 \\
\hline A4 & 0.9481 \\
\hline A5 & 0.9481 \\
\hline A6 & 0.9481 \\
\hline A7 & 0.9481 \\
\hline A8 & 0.9481 \\
\hline A9 & 0.9481 \\
\hline A10 & 0.9481 \\
\hline A11 & \\
\hline A12 & \\
\hline
\end{tabular}

Table 5: Optimized From Dataset2

\begin{tabular}{|l|r|}
\hline \multicolumn{2}{|c|}{ tmax2 } \\
\hline itemset & percentage \\
\hline \hline A1 & 0.94 \\
\hline A2 & 0.88 \\
\hline A3 & 0.87 \\
\hline A4 & 0.83 \\
\hline A5 & 0.82 \\
\hline A6 & 0.81 \\
\hline A7 & 0.8 \\
\hline A8 & 0.79 \\
\hline A9 & 0.775 \\
\hline A10 & 0.775 \\
\hline A11 & \\
\hline A12 & \\
\hline
\end{tabular}

Table 6: Optimized From Dataset3

\begin{tabular}{|l|r|}
\hline \multicolumn{2}{|c|}{ tmax3 } \\
\hline itemset & percentage \\
\hline \hline A1 & 0.9511 \\
\hline A2 & 0.9511 \\
\hline A3 & 0.9511 \\
\hline A4 & 0.9511 \\
\hline A5 & 0.9511 \\
\hline A6 & 0.9511 \\
\hline A7 & 0.9511 \\
\hline A8 & 0.9511 \\
\hline A10 & 0.9511 \\
\hline A11 & \\
\hline A12 & \\
\hline
\end{tabular}

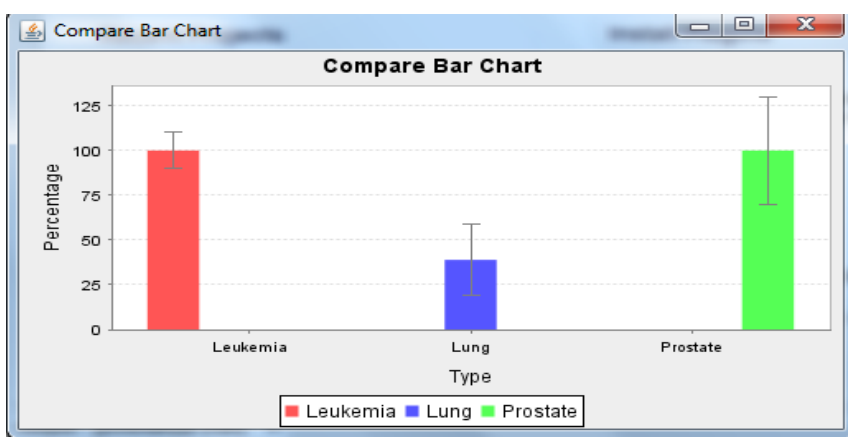

Fig 2: Classification Accuracy

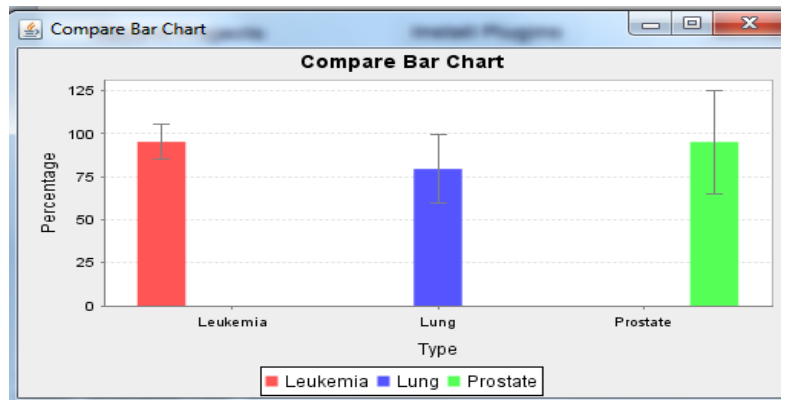

Figure 3: Overall Detection Accuracy

Table 7: Classification Accuracy Table

\begin{tabular}{|l|l|l|l|l|}
\hline NamelTechnique & SVM & $\begin{array}{l}\text { Naïve } \\
\text { Bayes }\end{array}$ & $\begin{array}{l}\text { Associative } \\
\text { Classifier }\end{array}$ & $\begin{array}{l}\text { ACO } \\
\text { based } \\
\text { classifier }\end{array}$ \\
\hline Leukemia & 95 & 93 & 96 & 100 \\
\hline Lung & 81 & 91 & 93 & 34 \\
\hline Prostate & 90 & 76 & 89 & 100 \\
\hline
\end{tabular}




\section{CONCLUSION}

A Hybrid framework is presented in this paper. The framework consists of association and ant colony optimization. Our work direction is in the way for better classification considering different dataset. It has been better explained with results also. Our results show the effectiveness of our approach. In future one can extend this work on other dataset also and check the effectiveness.

\section{ACKNOWLEDGMENTS}

I take this opportunity to thanks Dr. Divakar Singh (HOD, CSE Department, BUIT Bhopal) for accepting me to work under his valuable guidance, closely supervising this work over the past few months and offering many innovative ideas and helpful suggestions. His valuable advice and support, in spite of his busy schedule have really been an inspiration and driving force for me. He has constantly enriched my raw ideas with his experience and knowledge.

I would like to give thanks to Dr. Anju Singh (Asst. Prof. IT) for her valuable guidance and motivation.I also thank Dr. D. C. Gupta, Director BUIT, BU, Bhopal for extending all the help and cooperation during my dissertation work.

I thank my almighty parents, staff members of CSE and colleagues, and friends for their physical and moral support without which this assignment would not be possible.

Last but not the least, I must express my heartfelt thanks to my husband Mr. Yatendra Singh for his valuable support for successful completion of the work and for their understanding, endless patience, cooperation and constant encouragement when it was most required.

\section{REFERENCES}

[1] T.R. Golub, D.K. Slonim, P. Tamayo, C. Huard, M. Gaasenbeek, J.P. Mesirov, H. Coller, M.L. Loh, J.R. Downing, M.A. Caligiuri, C.D. Bloomfield, and E.S. Lander, "Molecular Classification of Cancer: Class Discovery and Class Prediction by Gene Expression Monitoring," Science, vol. 286, no. 5439, pp. 531-537, 1999.

[2] L. Li, C.R. Weinberg, T.A. Darden, and L.G. Pedersen, "Gene Selection for Sample Classification Based on Gene Expression Data: Study of Sensitivity to Choice of Parameters of the GA/ KNN Method," Bioinformatics, vol. 17, no. 12, pp. 1131-1142, 2001.

[3] T.S. Furey, N. Cristianini, N. Duffy, D.W. Bednarski, M. Schummer, and D. Haussler, "Support Vector Machine Classification and Validation of Cancer Tissue Samples Using Microarray Expression Data," Bioinformatics, vol. 16, no. 10, pp. 906-914, 2000.

[4] M.M. Xiong, L. Jin, W. Li, and E. Boerwinkle, "Tumor Classification Using Gene Expression Profiles," Biotechniques, vol. 29, pp. 1264-1270, 2000.

[5] Y. Wang, I.V. Tetko, M.A. Hall, E. Frank, A. Facius, K.F.X. Mayer, and H.W. Mewes, "Gene Selection from Microarray Data for Cancer Classification-A Machine Learning Approach," Computational Biology and Chemistry, vol. 29, no. 1, pp. 37-46, 2005.

[6] M. Xiong, X. Fang, and J. Zhao, "Biomarker Identification by Feature Wrappers," Genome Research, vol. 11, pp. 1878-1887, 2001.

[7] Yukyee Leung; Yeungsam Hung, "A Multiple-FilterMultiple-Wrapper Approach to Gene Selection and Microarray Data Classification," Computational Biology and Bioinformatics, IEEE/ACM Transactions on. vol.7, no.1, pp.108,117, Jan.-March 2010.

[8] JogendraKushwah, Divakar Singh,"Classification of Cancer Gene Selection Using Random Forest and Neural Network Based Ensemble Classifier",International Journal of Advanced Computer Research (IJACR), Volume-3 Number-2 Issue-10 June-2013.

[9] Dubey, A.K.; Dubey, A.K.; Agarwal, V.; Khandagre, Y., "Knowledge discovery with a subset-superset approach for Mining Heterogeneous Data with dynamic support," Software Engineering (CONSEG), 2012 CSI Sixth International Conference on , vol., no., pp.1,6, 5-7 Sept. 2012.

[10] Sachinsohra, NarendraRathod, "An Improved Single and Multiple association Approach for Mining Medical Databases",International Journal of Advanced Computer Research (IJACR), Volume 2, Number 2,June 2012.

[11] O. Cordón, F. Gomide, F. Herrera, F. Hoffmann, and L. Magdalena, "Ten years of genetic fuzzy systems: current framework and new trends," Fuzzy Sets Syst., vol. 141, pp. 5-31, 2004.

[12] F. Hoffmann, "Combining boosting and evolutionary algorithms for learning of fuzzy classification rules," Fuzzy Sets Syst., vol. 14, pp. 47-58, 2004.

[13] Dubey, A.K.; Shandilya, S.K., "A comprehensive survey of grid computing mechanism in J2ME for effective mobile computing techniques," Industrial and Information Systems (ICIIS), 2010 International Conference on , vol., no., pp.207,212, July 29 2010-Aug. 12010.

[14] Dubey, A.K.; Shandilya, S.K., "Exploiting Need of Data Mining Services in Mobile Computing Environments," Computational Intelligence and Communication Networks (CICN), 2010 International Conference on , vol., no., pp.409,414, 26-28 Nov. 2010

[15] Anshuman Singh Sadh, NitinShukla," Association Rules Optimization: A Survey", International Journal of Advanced Computer Research (IJACR), Volume-3 Number-1 Issue-9 March-2013.

[16] Anshuman Singh Sadh, NitinShukla, "Apriori and Ant Colony Optimization of Association Rules",International Journal of Advanced Computer Research (IJACR), Volume-3 Number-2 Issue-10 June-2013.

[17] Blaschke C., Oliveros, J.C. and Valencia, A., "Mining [31] Patrick C. H. Ma and Keith C. C. Chan," Incremental Fuzzy Mining of Gene Expression Data for Gene Function Prediction", IEEE Transactions on Biomedical Engineering, VOL. 58, NO. 5, MAY 2011.

[18] Debahuti Mishra and BarnaliSahu, "A Signal-to-noise Classification Model for Identification of Differentially Expressed Genes from Gene Expression Data", IEEE 2011.

[19] PradiptaMaji and Chandra Das," Relevant and Significant Supervised Gene Clusters for Microarray Cancer Classification", IEEE Transactions on Nano bioscience, Vol. 11, No. 2, June 2012.

[20] SmrutiRekha Das, Pradeepta Kumar Panigrahi, Kaberi Das and Debahuti Mishra, "Improving RBF Kernel Function of Support Vector Machine using Particle 
Swarm Optimization", International Journal of Advanced Computer Research (IJACR) Volume-2 Number-4 Issue7 December-2012.

[21] Jian-Bo Yang and Chong-Jin Ong,"An Effective Feature Selection Method via Mutual Information Estimation",IEEE Transactions on Systems, Man, and Cybernetics-Part B: Cybernetics, Vol. 42, No. 6, December 2012.
[22] Xiao Zhang, AichenLi,You Zhang, Yongpeng Xiao,"Validity of Cluster Technique for Genome Expression Data", IEEE 2012.

[23] Shang Gao, Omar Addam, AlaQabaja, AbdallahElSheikh, Omar Zarour, Mohamad Nagi,FlourisTriant,Wadhah Almansoori, Omer Sair, TanselOzyer, JiaZeng, JonRokne, RedaAlhajj "Robust Integrated Framework for Effective Feature Selection and Fuzzy Sets Syst., vol. 141, pp. 5-31, 2004 Sample Classification and Its Application to Gene Expression Data Analysis", IEEE 2012. 